\title{
Hábitos alimentarios de quironómidos (Diptera: Chironomidae) en lagos del páramo de Chingaza, Colombia
}

\author{
Yulibey Caleño Ruiz ${ }^{1}$, Carlos Alberto Rivera-Rondon ${ }^{1} \&$ Hernando Ovalle ${ }^{2}$ \\ 1. Laboratorio de Limnología, UNESIS, Departamento de Biología, Pontificia Universidad Javeriana, Bogotá, \\ Colombia; ycaleno@javeriana.edu.co, crivera@javeriana.edu.co \\ 2. Dirección de Investigación e Innovación Ambiental - Corporación Autónoma Regional de Cundinamarca, CAR, \\ Bogotá, Colombia; dracontos@gmail.com
}

Recibido 09-VIII-2017. Corregido 30-X-2017. Aceptado 17-XI-2017.

\begin{abstract}
Feeding habits of chironomids (Diptera: Chironomidae) of paramo lakes, Chingaza, Colombia. Tropical high mountain lakes show unique environmental conditions where chironomids play an important role in ecosystem functioning. The characteristics of these environments could favor diet overlap and therefore a high interspecific competition. This study described the dietary habits of chironomid genera, identified whether the dietary habits were specialized or generalist, and analyzed the diet overlap in the genera. Chironomidae larvae were collected from four lakes of the Chingaza paramo during the dry season, between April and May of 2016. The feeding habits of larvae were evaluated by analyzing gut contents following standard methods. Each genus was assigned to trophic guilds (carnivore, detritivore and algivore) and the diet overlap was estimated using the Pianka's index. A total of 1003 individuals were collected and nine genera were identified. Larvae consumed mainly fine particulate organic matter (FPOM), algae, macrophyte fragments, macroinvertebrates, and animal tissues. FPOM was the main feeding resource of detritivores. The analysis of diets showed a high affinity of each genus for a single trophic guild and most of the genera were generalist in the use of resources. For all lakes, high levels of diet overlap were observed among genera and trophic guilds, mainly among detritivores. Our results suggested that Chironomidae larvae of these lakes presented well differentiated trophic habits, and showed a moderate diet overlap within detritivores and carnivores. Rev. Biol. Trop. 66(1): 136-148. Epub 2018 March 01.
\end{abstract}

Key words: non-biting midges; gut contents; trophic guilds; Chironominae; Orthocladiinae; Tanypodinae.

Los páramos andinos son ecosistemas de alta montaña, de origen glacial y con una gran variedad de lagunas, turberas y pastizales húmedos entremezclados con matorrales y fragmentos forestales de baja altura (Buytaert et al., 2006). Son ecosistemas distribuidos heterogéneamente en los picos de las montañas de los Andes del Norte que durante el pleistoceno fueron moldeados físicamente por glaciaciones sucesivas (Van der Hammen \& Cleef, 1986). El origen y ubicación de los páramos permite que estén sujetos a condiciones ambientales particulares como son la alta radiación solar, cambios bruscos de temperatura durante el día y baja temperatura en la noche, $\mathrm{pH}$ ácidos, suelos ricos en materia orgánica (andisoles) y alta humedad. Estos factores son determinantes en la distribución de especies propias del páramo por lo cual se consideran estos ecosistemas como hotspots de biodiversidad global (Madriñán, Cortés, \& Richardson, 2013).

Los lagos de montaña tropical presentan condiciones limnológicas diferentes a los de zonas templadas. Pese a que la temperatura rara vez supera los $15^{\circ} \mathrm{C}$, son considerados lagos de aguas cálidas, reciben alta radiación solar durante todo el año y el balance de calor del cuerpo de agua está dado por la radiación diaria y el enfriamiento nocturno, con una mezcla convectiva nocturna en los más someros y atelomixis en los más profundos (Gunkel \& Casallas, 2002). Por otro lado, la materia 
orgánica proveniente de las macrófitas acuáticas presenta una descomposición lenta y es el principal aporte de detritus y materia orgánica fina, específicamente en la zona litoral donde se presenta una mayor densidad de macrófitas (Schmidt-Mumm \& Ríos, 2012).

La diversidad de macrófitas y los procesos de descomposición propician una mayor heterogeneidad de microambientes y una gran oferta alimenticia para los macroinvertebrados acuáticos (Lan et al., 2012). Dentro de este grupo, se encuentran larvas de la familia Chironomidae, consideradas importantes por su riqueza taxonómica y abundancia en lagos de alta montaña (Lods-Crozet, Oertli, \& Robinson, 2012; Sanchez-Hernandez, Cobo, \& Amundsen, 2015). Las larvas de quironómidos se encuentran distribuidas en las zonas de litoral, sublitoral y en los sedimentos de la zona profunda (Pinder, 1995). Cumplen un rol fundamental en el procesamiento de los diferentes tipos de materia orgánica (gruesa y fina), debido a la variedad morfo-comportamental para la adquisición del alimento, además de ser un vínculo entre productores (fitoplancton y algas bentónicas) y consumidores secundarios (Berg, 1995; Tokeshi, 1995; Silva, Ruiz, Bochini, \& Moreira, 2008).

El éxito de adaptación de los quironómidos a diferentes hábitats es debido al amplio límite de exploración trófica y aspectos microespaciales del ambiente (Dimitriadis \& Cranston, 2007; Butakka, Gomes, \& Takeda, 2014b). Las larvas de quironómidos son consideradas oportunistas omnívoras, que ingieren una gran variedad de alimentos (Cummins \& Klug, 1979). En general, se reporta un bajo grado de selectividad en su dieta, siendo clasificadas como generalistas (Berg, 1995; Henriques-Oliveira, Nessimian, \& Dorvillé, 2003; Sanseverino \& Nessimian, 2008). Estas consumen diferentes tipos de alimento como: algas, macrófitas, restos de madera, macroinvertebrados, detritos y microorganismos asociados (Berg, 1995). Mediante el análisis de contenido estomacal pueden designarse a distintos gremios tróficos: carnívoros, detritívoros, alguívoros, perifitívoros y omnívoros (Tomanova, Goitia, \& Helesic,
2006; Ceneviva-Bastos, Boracini, Mei, Conceicão, \& Casatti, 2017). Para el caso de lagos de alta montaña, se ha reportado dominancia de macroinvertebrados detritívoros y predadores (Sanchez-Hernandez et al., 2015).

La segregación espacial en hábitats disponibles para las diferentes especies puede conducir al solapamiento de nicho y por ende a competencia interespecífica (Schmid-Araya \& Schmid, 1995). La competencia por recursos entre larvas de Chironomidae puede limitar especies con nichos semejantes (Butakka, Ragonha, \& Takeda, 2014c). Estos aspectos no han sido investigados en lagunas de páramo. Este estudio busca i) describir los hábitos alimentarios de géneros de la familia Chironomidae en lagos del páramo de Chingaza, ii) establecer si tienen hábitos tróficos especializados o generalistas y iii) determinar el potencial grado de solapamiento en el uso de recursos entre géneros y gremios tróficos. Dadas las condiciones ambientales de los lagos de páramo y la heterogeneidad del hábitat, se presume que los quironómidos tendrían una oferta de distintos tipos de recurso que permitiría el desarrollo de géneros con diferentes hábitos tróficos y un bajo grado de solapamiento en el uso de los recursos alimenticios.

\section{MATERIALES Y MÉTODOS}

Área de estudio: Este estudio se desarrolló en cuatro lagos de páramo ubicados en el Parque Nacional Natural Chingaza, en jurisdicción de los departamentos de Cundinamarca y Meta, Colombia. Se estudiaron los lagos Buitrago, Chingaza, Siecha y Verde (Fig. 1). Estos fueron seleccionados tomando en cuenta criterios como altitud, extensión y diversidad de hábitats de la zona litoral. El parque se encuentra en la cordillera oriental de los andes colombianos, ocupa una extensión terrestre de 76600 ha y presenta temperaturas medias entre 4 y $20^{\circ} \mathrm{C}$, abarcando ecosistemas de páramo y bosque altoandino (Millán, 2016). Los lagos se encuentran altitudinalmente entre 3200 y $3600 \mathrm{msnm}$; en su zona litoral se observan rocas, raíces de plantas terrestres, detritos, 


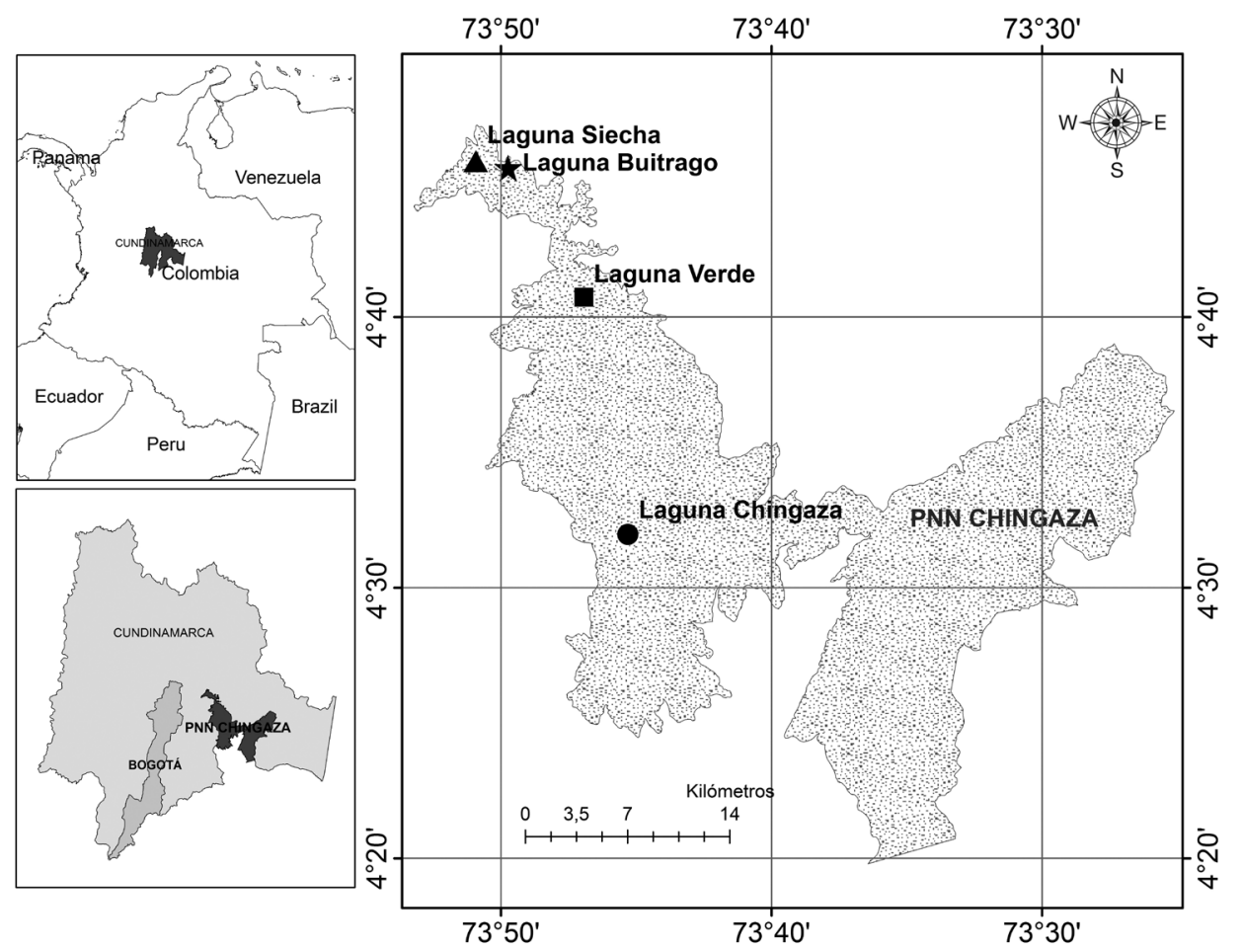

Fig. 1. Ubicación geográfica de los lagos estudiados en el Parque Nacional Natural Chingaza.

Fig. l. Geographic location of the studied lakes in Chingaza National Park.

sedimento y plantas acuáticas principalmente de los géneros Myriophyllum, Potamogeton, Elatine, Isoetes y Sphagnum.

Caracterización de lagos: En cada lago se realizó una descripción de la zona litoral a partir de la cual se identificaron los diferentes tipos de sustratos. Asimismo, se tomaron muestras de agua para analizar las concentraciones de nitrógeno total, fósforo total y carbono orgánico total. Las muestras fueron colectadas y analizadas siguiendo las recomendaciones y métodos de APHA, AWWA y WEF (2012). En campo se midió la temperatura, el $\mathrm{pH}$, la conductividad y el oxígeno usando una sonda YSI 556.

Muestreo de macroinvertebrados: $\mathrm{Se}$ realizó una única campaña de muestreo en cada lago entre abril y mayo de 2016, periodo que corresponde con el final del periodo de sequía de la zona. El muestreo se desarrolló en este periodo para evitar el efecto que tienen las lluvias en la perturbación de los sustratos del litoral. Durante el periodo de lluvias los niveles de estos lagos pueden presentar cambios, sumergiendo zonas circundantes cubiertas por vegetación del litoral. Durante el final del periodo de sequía se presenta una estabilidad en las condiciones hidrológicas y químicas de los ecosistemas que no solo garantiza una mejor representatividad del muestreo, sino que permite estudiar comunidades que han tenido un tiempo más largo para estructurarse.

En cada lago, se recolectaron muestras de diferentes sustratos de la zona litoral y sublitoral: rocoso, macrófitas (vegetación sumergida, flotante y emergente), raíces de plantas terrestres, detritos y sedimento. Desde un bote, se ubicó un cuadrante de $0.25 \mathrm{~m}^{2}$ en cada sustrato y con una red $\mathrm{D}$ de $250 \mu \mathrm{m}$ de apertura de poro se realizó durante cinco minutos la colecta 
de organismos. Este procedimiento se repitió tres veces por cada tipo de sustrato en diferentes zonas del lago. Adicionalmente, se tomó una muestra de sedimento de la zona profunda de cada lago mediante una draga Ponar de 200 $\mathrm{cm}^{2}$ de área efectiva de muestreo.

Las muestras fueron preservadas en campo con solución Kahle (Alcohol $95 \%$, formol $40 \%$, ácido acético glacial y agua destilada, en la proporción 15:6:2:30, respectivamente). Esta solución fija el tejido, lo hace más flexible y las estructuras internas, tales como el contenido intestinal y el tracto digestivo son más fáciles de disectar (Rosi-Marshall, Wellard, Hall, \& Vallis, 2016). En el laboratorio de limnología de la Pontificia Universidad Javeriana, las muestras fueron lavadas utilizando tamices de $1 \mathrm{~mm}, 250 \mu \mathrm{m}$ y $30 \mu \mathrm{m}$ de tamaño de poro, procediendo con una revisión manual en un estereoscopio Olympus SZ61, a modo de separar los quironómidos de la materia orgánica y recolectar larvas de diferentes estadios (Butakka et al., 2014c). Para el análisis no se separaron las larvas de cada género por estadios. Los especímenes fueron transferidos a viales de vidrio con etanol al $95 \%$ para su preservación a largo plazo, y su inclusión en las colecciones limnológicas del Museo de Historia Natural Lorenzo Uribe, de la Pontificia Universidad Javeriana con números de catálogo MPUJ-M3785 al MPUJ-M4241.

Determinación de géneros: Los quironómidos fueron separados de los demás grupos de macroinvertebrados y se seleccionaron de dos a cuatro individuos por cada morfotipo para la determinación a género. Las capsulas cefálicas o cabezas se introdujeron en $\mathrm{KOH}$ al $10 \%$ durante 48 horas para aclarar estructuras internas y externas, posteriormente fueron sumergidas en ácido acético glacial, dejando en exposición durante aproximadamente diez minutos (Pinho, 2008). El resto del cuerpo se preservó en etanol al $95 \%$ para conservar estructuras como parápodos, procercos, túbulos ventrales o túbulos anales, requeridas para la identificación. Se realizó el montaje de capsulas cefálicas en láminas de vidrio con glicerina, ubicando la cápsula en vista ventral. Para conservar los montajes por varios días se colocó un cubreobjetos y se selló con esmalte. Las cabezas se observaron en un microscopio óptico (1000 y 400 aumentos) y la identificación se realizó usando claves especializadas (Ospina, Riss, \& Ruiz, 1999; Ruiz-Moreno, Ospina-Torres, \& Riss, 2000; Ruiz-Moreno, Ospina-Torres, Gómez-Sierra, \& Riss, 2000; Epler, 2001; Prat, Rieradevall, Acosta, \& Villamarín, 2011).

Contenidos estomacales: De acuerdo con la disponibilidad de especímenes, se seleccionaron hasta diez individuos del mismo género, como una cantidad representativa por cada taxón para el análisis de variabilidad en la dieta (Henriques-Oliveira et al., 2003; Tomanova et al., 2006; Rosi-Marshall et al., 2016). Se extrajo el tracto digestivo y el contenido estomacal se montó en una lámina de vidrio con glicerina, se colocó un cubreobjetos y se selló con esmalte. Los ítems alimentarios se asignaron según Tomanova et al. (2006) como: material mineral o sedimento, materia orgánica particulada gruesa MOPG $>1 \mathrm{~mm}$, materia orgánica particulada fina MOPF $<1 \mathrm{~mm}$, algas, macrófitas, tejido animal, zooplancton, macroinvertebrados y hongos. Para estimar la proporción de cada ítem alimentario, se escogieron diez campos al azar de cada lámina y se midió el área ocupada por cada ítem a 400 aumentos usando un microscopio Carl Zeiss Axio Imager A2 con cámara integrada Lumenera's Infinity 3 y el programa Infinity Analize. Este procedimiento se repitió hasta revisar los tractos digestivos de 51 especímenes, contando 505 campos.

Con el objeto de ordenar y relacionar los items alimentarios y los géneros, se realizó un Análisis de Escalamiento Multidimensional no Métrico (NMDS) usando la distancia de BrayCurtis en el programa R (R Core Team, 2016) y el paquete Vegan (Oksanen et al., 2015).

Gremios tróficos: La asignación de cada género a su respectivo nivel trófico se basó en el análisis de contenidos estomacales. Se utilizó una escala categórica para determinar mediante la proporción de los ítems alimentarios 
la afinidad al gremio trófico; sin afinidad (0), poca afinidad (1), media afinidad (2) y fuerte afinidad (3) (Tomanova et al., 2006). Adicionalmente, otros estudios de hábitos alimentarios en quironómidos fueron revisados para comparar y confirmar las designaciones realizadas (Henriques-Oliveira et al., 2003; Sanseverino \& Nessimian, 2008; Silva et al., 2008; Silva, Mayer, Biscalquini, \& Silveira, 2009; Galizzi, Zilli, \& Marchese, 2012; Butakka, Grzybkowska, Pinha, \& Takeda, 2014a).

Solapamiento en la dieta: Para estimar el solapamiento en la dieta de quironómidos se utilizó el índice de Pianka (Krebs, 2014). Se estimó el índice $\left(\mathrm{O}_{\mathrm{H}}\right)$ para todos los pares de géneros y gremios tróficos de cada laguna así:

$$
O_{H}=\frac{\sum_{\mathrm{i}}^{\mathrm{n}} \mathrm{p}_{\mathrm{ij}} \mathrm{p}_{\mathrm{ik}}}{\sqrt{\sum_{\mathrm{i}}^{\mathrm{n}} \mathrm{p}_{\mathrm{ij}}^{2}+\sum_{i}^{\mathrm{n}} \mathrm{p}_{\mathrm{ik}}^{2}}}
$$

Donde,

$\mathrm{p}_{\mathrm{ij}}=$ proporción del recurso $\mathrm{i}$ del total de recursos utilizados por especies $\mathrm{j}$

$\mathrm{p}_{\mathrm{ik}}=$ proporción del recurso $\mathrm{i}$ del total de recursos utilizados por especies $\mathrm{k}$

$\mathrm{n}=$ número total de recursos $(\mathrm{i}=1,2,3, \ldots \mathrm{n})$.

Este índice varía de cero a uno, con un valor de cero cuando no hay recursos utilizados en común y un valor de uno cuando existe un solapamiento completo. La significancia del solapamiento se valoró con un test de modelos nulos usando el paquete EcoSimR de R (Gote1li, Hart, \& Ellison, 2015; R Core Team, 2016). En el modelo nulo se usaron 5000 aleatorizaciones de los recursos, reemplazando los datos con valores aleatorios uniformes. Dado que no se extrajo el tracto digestivo de la totalidad de larvas de los cuatro lagos, se utilizó la información de la dieta de cada género para estimar el solapamiento por cada lago.

\section{RESULTADOS}

Parámetros físicos y químicos: Los lagos estudiados se caracterizaron por presentar aguas oxigenadas $\left(6.3-6.5 \mathrm{mg} \mathrm{O}_{2} / \mathrm{L}\right)$ y bajos valores de conductividad (Cuadro 1). El valor de conductividad más alto se presentó en el lago Chingaza $(30 \mu \mathrm{S} / \mathrm{cm})$. Todos los lagos presentaron aguas ácidas con valores más bajos en Siecha y Buitrago (5.1 y 5.7, respectivamente). De acuerdo con los valores de fósforo, que estuvieron entre $<0.003$ y $0.055 \mathrm{mg} / \mathrm{L}$, todos los lagos pueden considerarse como oligotróficos.

Larvas de quironómidos: Se colectaron 1003 individuos agrupados en nueve géneros y tres subfamilias, Chironominae: Chironomus (Chi), Dicrotendipes (Dic) y Tanytarsus (Tan);

CUADRO 1

Características físicas y químicas de los lagos estudiados en el páramo de Chingaza, Colombia

TABLE 1

Physical and chemical characteristics of the studied lakes in Chingaza paramo, Colombia

\begin{tabular}{lcccc}
\multicolumn{1}{c}{ Parámetro } & Buitrago & Chingaza & Siecha & Verde \\
Altura $(\mathrm{msnm})$ & 3603 & 3224 & 3590 & 3538 \\
Área $(\mathrm{ha})$ & 1.14 & 104.8 & 6.0 & 2.99 \\
Prof. máx. $(\mathrm{m})$ & 0.7 & 24.8 & 12.3 & 11.3 \\
Temperatura $\left({ }^{\circ} \mathrm{C}\right)$ & 11.5 & 13.3 & 5.1 & 14.1 \\
$\mathrm{pH}$ & 5.7 & 6.8 & 6.5 & 6.7 \\
Oxígeno disuelto $(\mathrm{mg} / \mathrm{L})$ & 6.5 & 6.5 & 3 & 6.3 \\
Conductividad $(\mu \mathrm{S} / \mathrm{cm})$ & 2 & 30 & 1.434 & 12 \\
Nitrógeno total $(\mathrm{mg} / \mathrm{L})$ & 1.620 & 1.385 & 0.008 & 0.006 \\
Fósforo total $(\mathrm{mg} / \mathrm{L})$ & 0.055 & $<0.003$ & 2.7 & 13 \\
Carbono orgánico total $(\mathrm{mg} / \mathrm{L})$ & 7.2 & 8.9 & & \\
\hline
\end{tabular}


Orthocladiinae: Metriocnemus (Met), Parametriocnemus (Par) y Pseudosmittia (Pse); Tanypodinae: Ablabesmyia (Abl), Alotanypus (Alo) y Labrundinia (Lab). El género Dicrotendipes fue dominante en el lago Chingaza y Siecha. El lago Siecha presentó la mayor riqueza de géneros y el lago Buitrago la mayor abundancia de individuos (Fig. 2A). Las macrófitas albergaron una mayor riqueza de géneros y abundancias más altas, seguido por los sustratos sedimentos y detritos. La menor riqueza y abundancia se presentó en raíces de vegetación y rocas (Fig. 2B).

Contenido estomacal: La MOPF, algas, fragmentos de macrófitas, macroinvertebrados y tejido animal, fueron las categorías alimentarias ingeridas por larvas de Chironomidae. No se encontró MOPG en ninguno de los individuos analizados. La MOPF y el tejido animal, fueron los ítems con mayor proporción y frecuencia en los contenidos estomacales. Mientras que las algas mostraron una baja proporción en la dieta de larvas (Cuadro 2).
En los géneros Chironomus, Dicrotendipes, Metriocnemus, Parametriocnemus, Pseudosmittia y Tanytarsus, el ítem alimentario más frecuente en los contenidos estomacales fue la MOPF (88.7 - $100 \%)$, seguido por una baja proporción de algas $(0.4-4.3 \%)$. Pseudosmittia presentó restos de plantas, pero en baja proporción (10\%). En cuanto a Ablabesmyia, Alotanypus y Labrundinia, los contenidos estomacales presentaron macroinvertebrados (quironómidos y ácaros) y tejido animal (restos de insectos y cladóceros) (Fig. 3 y Cuadro 2).

Gremios tróficos: De acuerdo con el análisis de contenido estomacal, los géneros Ablabesmyia, Alotanypus y Labrundinia se clasificaron como carnívoros. Chironomus, Dicrotendipes, Parametriocnemus y Pseudosmittia fueron clasificados como detritívoros/ alguívoros, mientras que Metriocnemus y Tanytarsus como detritívoros. El grupo dietario más frecuente fue de los detritívoros. Los géneros evaluados presentaron hábitos tróficos
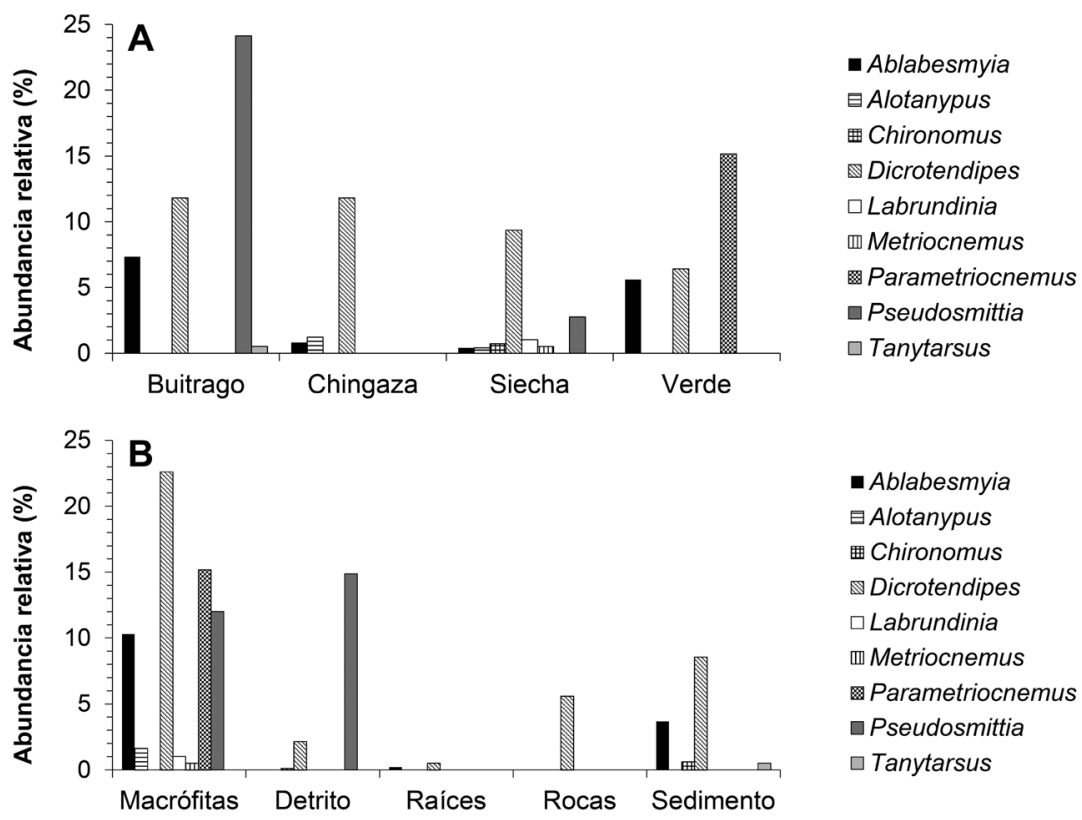

Fig. 2. Géneros de quironómidos presentes en lagos del páramo de Chingaza. Se presenta la abundancia relativa de los géneros en cada lago (A) y sustratos de la zona litoral (B).

Fig. 2. Chironomids genera found in lakes of Chingaza paramo. The relative abundance of genera in lakes (A) and littoral substrate (B) are shown. 
CUADRO 2

Abundancia relativa de ítems alimentarios en el contenido estomacal de géneros de Chironomidae

TABLE 2

Relative abundance of food items ingested by Chironomidae in Chingaza

\begin{tabular}{|c|c|c|c|c|c|}
\hline Género & Alg & $\mathrm{MaFi}$ & MaIn & MOPF & TA \\
\hline Ablabesmyia & & & $16.0(0.3)$ & & $84.0(0.8)$ \\
\hline Alotanypus & & & $33.3(0.3)$ & & $66.7(0.6)$ \\
\hline Chironomus & $2.4(0.6)$ & & & $97.6(1)$ & \\
\hline Dicrotendipes & $4.3(0.9)$ & & & $95.7(1)$ & \\
\hline Labrundinia & & & & & $100(1)$ \\
\hline Metriocnemus & & & & $100(1)$ & \\
\hline Parametriocnemus & $0.4(0.8)$ & & & $99.6(1)$ & \\
\hline Pseudosmittia & $1.3(0.4)$ & $10.0(0.1)$ & & $88.7(0.9)$ & \\
\hline Tanytarsus & & & & $100(1)$ & \\
\hline
\end{tabular}

* En paréntesis se presenta la frecuencia de individuos de cada género en la que el respectivo ítem fue encontrado. Algas (Alg), Macrófitas (MaFi), Macroinvertebrados (MaIn), Materia Orgánica Particulada Fina (MOPF) y Tejido Animal (TA).

* In parentheses is the frequency of individuals of each genus, in which the respective item was found. Algae (Alg), Macrophytes (MaFi), Macroinvertebrates (MaIn), Fine Particulate Organic Matter (MOPF), and animal tissue (TA).

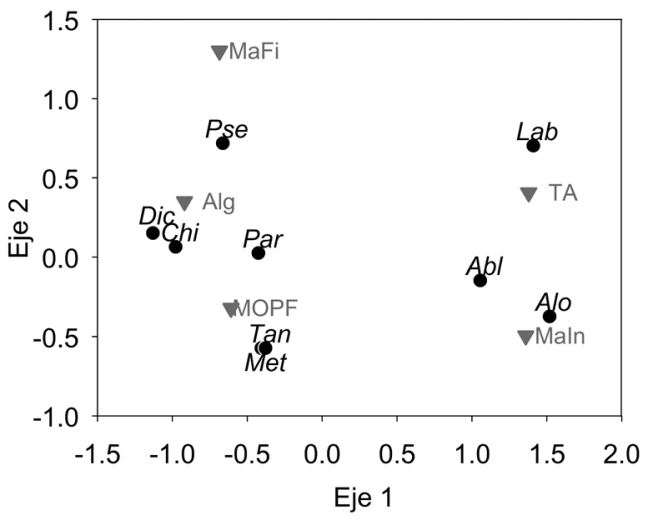

Fig. 3. Biplot del Escalamiento Multidimensional no Métrico realizado para ítems alimentarios ingeridos por géneros de Chironomidae en lagos del páramo de Chingaza. Algas (Alg), Macrófitas (MaFi), Macroinvertebrados (MaIn), Materia Orgánica Particulada Fina (MOPF) y Tejido animal (TA). Ablabesmyia (Abl), Alotanypus (Alo), Chironomus (Chi), Dicrotendipes (Dic), Labrundinia (Lab), Metriocnemus (Met), Parametriocnemus (Par), Pseudosmittia (Pse) y Tanytarsus (Tan).

Fig. 3. Biplot of a Non-Metric Multidimensional Scaling applied for food items ingested by Chironomidae genera in the lakes of the Chingaza paramo. Algae (Alg), Macrophytes (MaFi), Macroinvertebrates (MaIn), Fine Particulate Organic Matter (MOPF), and animal tissue (TA). Ablabesmyia (Abl), Alotanypus (Alo), Chironomus (Chi), Dicrotendipes (Dic), Labrundinia (Lab), Metriocnemus (Met), Parametriocnemus (Par), Pseudosmittia (Pse) y Tanytarsus (Tan). bien diferenciables con alta afinidad a un único grupo trófico (Fig. 4).

Solapamiento de dietas: El índice de Pianka mostró solapamiento entre géneros y grupos dietarios en todas las lagunas evaluadas, pero fue más evidente en la laguna

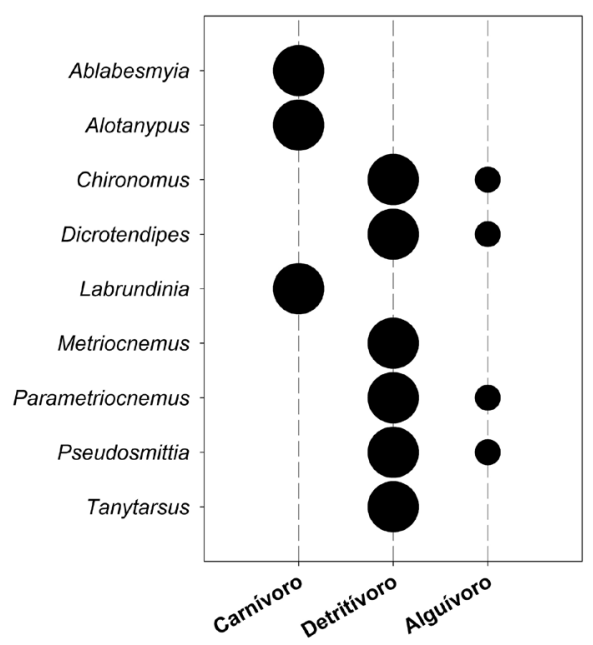

Fig. 4. Asignación de los géneros estudiados a gremios tróficos. Círculos grandes=alta afinidad, círculos pequeños $=$ baja afinidad.

Fig. 4. Assignment of studied genera to trophic guilds. Large circles=high affinity, small circles $=$ low affinity. 
CUADRO 3

Índice de solapamiento de Pianka entre géneros y gremios tróficos en cada uno de los lagos estudiados

TABLE 3

Pianka overlap index for genera and trophic guilds in each lake

\begin{tabular}{|c|c|c|c|c|}
\hline Lago & Géneros & Gremio trófico & Índice de Pianka & p-valor \\
\hline \multirow[t]{3}{*}{ Chingaza } & Ablabesmyia - Alotanypus & Car - Car & 0.9885 & 0.0034 \\
\hline & Ablabesmyia - Dicrotendipes & Car - Det/Alg & 0 & - \\
\hline & Alotanypus - Dicrotendipes & Car - Det/Alg & 0 & - \\
\hline \multirow[t]{6}{*}{ Buitrago } & Ablabesmyia - Dicrotendipes & Car - Det/Alg & 0 & - \\
\hline & Ablabesmyia - Pseudosmittia & Car - Det/Alg & 0 & - \\
\hline & Ablabesmyia - Tanytarsus & Car - Det & 0 & - \\
\hline & Dicrotendipes - Pseudosmittia & Det/Alg - Det/Alg & 0.99377 & 0.0014 \\
\hline & Dicrotendipes - Tanytarsus & Det/Alg - Det & 0.99983 & $<0.0001$ \\
\hline & Pseudosmittia - Tanytarsus & Det/Alg - Det & 0.9942 & 0.0012 \\
\hline \multirow[t]{15}{*}{ Siecha } & Ablabesmyia - Alotanypus & Car - Car & 0.9885 & 0.0034 \\
\hline & Ablabesmyia - Chironomus & Car - Det/Alg & 0 & - \\
\hline & Ablabesmyia - Dicrotendipes & Car - Det/Alg & 0 & - \\
\hline & Ablabesmyia - Labrundinia & Car - Car & 0.98801 & 0.0054 \\
\hline & Alotanypus - Chironomus & Car - Det/Alg & 0 & - \\
\hline & Alotanypus - Dicrotendipes & Car - Det/Alg & 0 & - \\
\hline & Alotanypus - Labrundinia & Car - Car & 0.9532 & 0.0530 \\
\hline & Alotanypus - Metriocnemus & Car - Det & 0 & - \\
\hline & Alotanypus - Pseudosmittia & Car - Det/Alg & 0 & - \\
\hline & Chironomus - Dicrotendipes & Det/Alg - Det/Alg & 0.9998 & 0.0006 \\
\hline & Chironomus - Labrundinia & Det/Alg - Car & 0 & - \\
\hline & Chironomus - Metriocnemus & Det/Alg - Det & 0.9998 & 0.0010 \\
\hline & Dicrotendipes - Labrundinia & Det/Alg - Car & 0 & - \\
\hline & Dicrotendipes - Metriocnemus & Det/Alg - Det & 0.9999 & 0.0002 \\
\hline & Labrundinia - Metriocnemus & Car - Det & 0 & - \\
\hline \multirow[t]{3}{*}{ Verde } & Ablabesmyia - Dicrotendipes & Car - Det/Alg & 0 & - \\
\hline & Ablabesmyia - Parametriocnemus & Car - Det/Alg & 0 & - \\
\hline & Dicrotendipes - Parametriocnemus & Det/Alg - Det/Alg & 0.9999 & 0.0002 \\
\hline
\end{tabular}

* Alg=Alguívoro, Car=Carnívoro y Det= Detritívoro.

* Alg=Algivore, Car=Carnivore y Det= Detritivore .

Siecha, con un mayor número de interacciones solapadas. Los valores del índice fueron altos, lo que indica un completo solapamiento en el uso de recursos. La ausencia de solapamiento también fue observada dentro de géneros y gremios tróficos, especialmente entre depredadores y otros grupos. Se observó solapamiento entre Chironomus, Dicrotendipes, Tanytarsus, Metriocnemus, Parametriocnemus y Pseudosmittia en la laguna Buitrago, Siecha y Verde. El número de interacciones de solapamiento fue mayor entre detritívoros. Dentro del grupo de depredadores se observó solapamiento en la laguna Siecha y Chingaza, concretamente entre Ablabesmyia, Alotanypus y Labrundinia (Cuadro 3). El análisis de solapamiento de todos los posibles pares de interacciones entre los géneros presentes en cada lago, mostró que en Buitrago el $50 \%$ de los posibles casos presentó solapamiento; en Chingaza y Verde un $33 \%$; mientras que en Siecha el $42 \%$ de las interacciones presentaron solapamiento. 


\section{DISCUSIÓN}

La composición de géneros de estos lagos es comúnmente reportada para ecosistemas acuáticos de montaña. Chironomus, Dicrotendipes, Tanytarsus, Parametriocnemus, Pseudosmittia, Ablabesmyia y Labrundinia, son géneros de amplia distribución altitudinal y han sido encontrados tanto en ecosistemas de zonas bajas como de alta montaña tropical (Henriques-Oliveira et al., 2003; Catalan et al., 2006; Sanseverino \& Nessimian, 2008; Silva et al., 2008; Prat, González-Trujillo, \& OspinaTorres, 2014; Butakka et al., 2014a; Butakka et al., 2014b). Alotanypus es un género reportado especialmente en lagos de alta montaña y ríos altoandinos de la región Neotropical (Prat et al., 2011; Prat et al., 2014; Silva, 2016). La riqueza de géneros encontrada en este estudio fue más alta que la reportada en otros estudios de lagos de páramo en Colombia (PosadaGarcía, Abril-Ramírez, \& Parra-Sánchez, 2008; Alba-Hincapié, González-Rey, \& Longo, 2016; Gómez, Salazar, \& Longo, 2016).

La MOPF fue la categoría alimentaria más frecuente en la mayoría de larvas de Chironomidae, como es comúnmente reportado para este grupo en el ámbito global (Berg, 1995; Henriques-Oliveira et al., 2003; Sanseverino \& Nessimian, 2008; Silva et al., 2008; Galizzi et al., 2012). Las algas también han sido reportadas como otro ítem presente en contenidos estomacales de quironómidos de la región Neotropical (Butakka, Ragonha, Train, Pinha, \& Takeda, 2016). No obstante, en las lagunas estudiadas su importancia fue menor al $5 \%$ y pese a su relativa alta frecuencia en las dietas, los bajos valores observados podrían corresponder con los esperados por consumo incidental.

Las larvas de Chironominae y Orthocladiinae mostraron variabilidad en su dieta acorde con lo reportado en la literatura (HenriquesOliveira et al., 2003; Sanseverino \& Nessimian, 2008). Por el contrario, los géneros de Tanypodinae se alimentaron exclusivamente de organismos como: macroinvertebrados, cladóceros e insectos terrestres. Las larvas de
Ablabesmyia tuvieron en sus estómagos únicamente restos de animales, ácaros y larvas de Dicrotendipes (Chironominae). En contenidos estomacales de Labrundinia se encontró fragmentos animales, y para el caso de Alotanypus, se encontraron restos animales y larvas de Tanypodinae indicando el canibalismo entre esta subfamilia. Otros estudios sugieren que los géneros Ablabesmyia y Labrundinia pueden usar una mayor variedad de fuentes alimenticias, reportando también detrito, polen y algas (Henriques-Oliveira et al., 2003; Butakka et al., 2014a). Sin embargo, no se encontraron estas categorías en las larvas analizadas.

Los resultados indican que la dieta de las larvas en estos lagos de páramo es menos diversificada, con respecto a lo reportado por otros estudios para Tanypodinae, Chironominae y Orthocladiinae (Henriques-Oliveira et al., 2003; Sanseverino \& Nessimian, 2008; Butakka et al., 2014a). La baja amplitud de la dieta puede estar relacionada con el tipo de recursos que el medio ofrece a los invertebrados. La abundante presencia de macrófitas en la zona litoral de lagos de montaña neotropicales (Schmidt-Mumm \& Ríos, 2012), promueve una alta acumulación de materia orgánica y una alta disponibilidad de carbono orgánico para las redes tróficas (Van Colen et al., 2017). Así mismo, la ausencia de quironómidos fragmentadores y la baja actividad microbiana en las condiciones ambientales del páramo, favorecen una baja tasa de descomposición (Posada-García et al., 2008; Meneses-Ortegón \& Herrera-Martínez, 2015). Estas condiciones pueden favorecer una baja riqueza de ítems en la dieta debido a la alta oferta de recursos de un mismo tipo (Mason, Mouillot, Lee, \& Wilson, 2005) y por lo tanto, la alta oferta de MOPF puede promover el desarrollo de comunidades pobremente diversificadas en grupos tróficos.

Si bien en las lagunas de páramo es evidente la oferta potencial del recurso alimenticio para los detritívoros, proveniente de la descomposición de las macrófitas, la disponibilidad en forma de MOPF está limitada por la baja riqueza y densidad de macroinvertebrados fragmentadores (Contreras \& Téllez, 2015), 
la lenta tasa de descomposición asociada con la baja temperatura y alta radiación ultravioleta que son factores de estrés que limitan la actividad microbiana (Zuñiga, Uribe, Torres, Cuero, \& Peña, 2013).

Se ha reportado a los quironómidos como un grupo generalista u oportunista (Berg, 1995; Henriques-Oliveira et al., 2003; Sanseverino \& Nessimian, 2008; Butakka et al., 2016). Aunque la MOPF constituye la principal fuente de alimento, son capaces de aprovechar otros recursos, por lo que el nivel de especialización esperado es muy bajo. Si bien algunos de los géneros fueron reconocidos como depredadores que se alimentaron exclusivamente de otros organismos, el análisis dietario no permitió definir si hay algún nivel de selectividad en las mismas, para definirlas como especialistas.

Dado que los valores de solapamiento fueron altos, se podría asumir que existe competencia potencial entre las especies de estos ambientes, no obstante, la similitud en la dieta entre diferentes taxones en un mismo nicho no necesariamente resulta en competencia entre estos, sino una relación de coexistencia cuando el recurso no es escaso en el hábitat, incluso en nichos parcialmente solapados (Butakka et al., 2014c). La estimación de la oferta alimenticia es difícil de evaluar en este tipo de ambientes, por lo que no es posible establecer la competencia efectiva entre géneros con las mismas dietas. En términos de las interacciones entre los géneros de cada lago estudiado, podría estimarse que el solapamiento en el uso de los recursos fue menor a un $50 \%$ de todas las posibles interacciones.

En este estudio concluimos que las larvas de quironómidos de lagos del páramo de Chingaza, tienen hábitos carnívoros, detritívoros y alguívoros. La MOPF es el principal tipo de alimento consumido por los detritívoros mientras que los depredadores consumen macroinvertebrados y zooplancton. Las larvas de Chironomidae presentan hábitos generalistas, con baja selectividad en su dieta y alta afinidad a un único gremio trófico. Se evidencia un moderado nivel de solapamiento trófico entre géneros y grupos dietarios, que es más evidente entre detritívoros. Este trabajo constituye una de las primeras aproximaciones al análisis de la dieta de quironómidos en lagunas de páramo. Los resultados obtenidos a partir del análisis de las dietas y del solapamiento, sugieren que las lagunas de páramos son ecosistemas fisionómicamente complejos, en donde una alta oferta de un tipo de recurso se relaciona con una baja diversidad de ítems consumidos por los quironómidos y una baja diversidad de gremios tróficos.

\section{AGRADECIMIENTOS}

A Colciencias por la vinculación de Yulibey Caleño cómo joven investigadora (2015). A la Pontificia Universidad Javeriana por la financiación de este estudio (proyecto "Influencia del hábitat sobre la estructura de los ensamblajes de diatomeas en lagos de montaña" SIAP6430). Al personal del Parque Nacional Natural Chingaza, por su colaboración durante el estudio. A los miembros del Laboratorio de Limnología, en especial a Wilmer Arguello y Jorge Jiménez por su apoyo en campo.

\section{RESUMEN}

Los lagos tropicales de alta montaña presentan condiciones ambientales únicas donde los quironómidos tienen un papel importante en el funcionamiento del ecosistema. Las características de estos ambientes podrían favorecer el solapamiento de dietas y por ende una alta competencia interespecífica. En este estudio se describen los hábitos alimentarios de géneros de quironómidos, se identifica si los hábitos alimenticios son especializados o generalistas y se analiza el solapamiento en la dieta de los géneros. Entre abril y mayo de 2016 se colectaron larvas de Chironomidae de cuatro lagos del páramo de Chingaza. Se evaluó la dieta de larvas, mediante el análisis de contenido estomacal. Se ubicó cada género en gremios tróficos (carnívoro, detritívoro y alguívoro) y se estimó el solapamiento de las dietas entre los géneros mediante el índice de Pianka. Se colectó un total de 1003 individuos y se identificaron nueve géneros. Las larvas consumieron principalmente materia orgánica particulada fina (MOPF), algas, fragmentos de macrófitas, macroinvertebrados y tejido animal. La MOPF fue la fuente principal de alimento de detritívoros. El análisis de dietas mostró una alta afinidad de cada género con un único gremio trófico y la mayor parte de los géneros son generalistas en el uso de recursos. En todos los lagos se 
observaron valores altos de solapamiento en la dieta entre los géneros y los gremios tróficos, principalmente entre los detritívoros. Los resultados sugieren que las larvas de Chironomidae presentan hábitos tróficos bien diferenciados, ocurriendo un moderado solapamiento de dietas entre géneros de detritívoros y carnívoros.

Palabras clave: larvas; contenido estomacal; gremios tróficos; Chironominae; Orthocladiinae; Tanypodinae.

\section{REFERENCIAS}

Alba-Hincapié, Á. M., González-Rey, G., \& Longo, M. (2016). Macroinvertebrados asociados a macrófitas en la laguna La Virginia, páramo Sumapaz, Colombia. Biota Colombiana, 17(2), 3-19.

APHA, AWWA, \& WEF. (2012). Standard Methods for examination of water and wastewater (22a ed.). Washington, D.C: American Public Health Association.

Berg, M. B. (1995). Larval food and feeding behaviour. En P. D. Armitage, P. Cranston, \& L. C. Pinder (Eds.), The Chironomidae: Biology and ecology of non-biting midges (1a ed., pp. 136-168). Netherlands:Springer.

Butakka, C. M. M., Ragonha, F. H., Train, S., Pinha, G. D., \& Takeda, A. M. (2016). Chironomidae feeding habits in different habitats from a Neotropical floodplain: exploring patterns in aquatic food webs. Brazilian Journal of Biology, 76(1), 117-125.

Butakka, C., Grzybkowska, M., Pinha, G., \& Takeda, A. (2014a). Habitats and trophic relationships of Chironomidae insect larvae from the Sepotuba River basin, Pantanal of Mato Grosso, Brazil. Brazilian Journal of Biology, 74(2), 395-407.

Butakka, C. M., Gomes, L. C., \& Takeda, A. (2014b). Taxonomic and numeric structure of Chironomidae (Diptera) in different habitats of a Neotropical floodplain. Iheringia, Série Zoologia, 104(3), 314-322.

Butakka, C. M. M., Ragonha, F. H., \& Takeda, A. M. (2014c). Chironomidae larvae (Diptera) of Neotropical floodplain: overlap niche in different habitats. Brazilian Journal of Biology, 74(2), 363-370.

Buytaert, W., Célleri, R., De Bièvre, B., Cisneros, F., Wyseure, G., Deckers, J., \& Hofstede, R. (2006). Human impact on the hydrology of the Andean páramos. Earth-Science Reviews, 79, 53-72.

Catalan, J., Camarero, L., Felip, M., Pla, S., Ventura, M., Buchaca, T., ... Díaz, D. (2006). High mountain lakes: extreme habitats and witnesses of environmental changes. Limnetica, 25(1-2), 551-584.

Ceneviva-Bastos, M., Boracini, D., Mei, R., Conceicão, P., \& Casatti, L. (2017). Trophic guilds of EPT (Ephemeroptera, Plecoptera and Trichoptera ) in three basins of the Brazilian Savanna. Limnologica, $63,11-17$.

Contreras, T. A., \& Téllez, D. C. (2015). Rasgos funcionales de macroinvertebrados acuáticos asociados a dos subsistemas de la laguna La Virginia, páramo de Sumapaz. Bogotá, Colombia: Fundación Universidad de Bogota Jorge Tadeo Lozano.

Cummins, K. W., \& Klug, M. J. (1979). Feeding ecology of stream invertebrates. Annual Review of Ecology and Systematics, 10(1), 147-172.

Dimitriadis, S., \& Cranston, P. S. (2007). From the mountains to the sea: assemblage structure and dynamics in Chironomidae (Insecta: Diptera) in the Clyde River estuarine gradient, New South Wales, SouthEastern Australia. Australian Journal of Entomology, 46, 188-197.

Epler, J. H. (2001). Identification manual for the larval Chironomidae (Diptera) of north and south carolina. (1a ed.). North Carolina, USA: North Carolina Department of Environment and Natural Resources Division of Water Quality.

Galizzi, M. C., Zilli, F., \& Marchese, M. (2012). Diet and functional feeding groups of Chironomidae (Diptera) in the Middle Paraná River floodplain (Argentina). Iheringia. Série Zoologia, 102(2), 117-121.

Gómez, S., Salazar, C., \& Longo, M. (2016). Diversidad y biomasa de macroinvertebrados asociados a cuatro tipos de sustratos en la laguna La Virginia, páramo Sumapaz, Colombia. Biota Colombiana, 17(2), 20-38.

Gotelli, N., Hart, E. M., \& Ellison, A. M. (2015). EcoSimR: Null model analysis for ecological data. R package version 0.1.0.

Gunkel, G., \& Casallas, J. (2002). Limnology of an equatorial high mountain lake - Lago San Pablo, Ecuador: The significance of deep diurnal mixing for lake productivity. Limnologica, 32, 33-43.

Henriques-Oliveira, A. L., Nessimian, J. L., \& Dorvillé, L. F. (2003). Feeding habits of chironomid larvae (Insecta: Diptera) from a stream in the Floresta da Tijuca, Rio de Janeiro, Brazil. Brazilian Journal of Biology, 63(2), 269-281.

Krebs, C. J. (2014). Niche measures and resource preferences. Ecological methodology, 3, 597-653.

Lan, Y., Cui, B., You, Z., Li, X., Han, Z., Zhang, Y., \& Zhang, Y. (2012). Litter decomposition of six macrophytes in a eutrophic shallow lake (Baiyangdian Lake, China). Clean - Soil, Air, Water, 40(10), 1159-1166.

Lods-Crozet, B., Oertli, B., \& Robinson, C. T. (2012). Long-term patterns of chironomid assemblages in a high elevation stream / lake network 
(Switzerland)-Implications to global change. Fauna norvegica, 31, 71-85.

Madriñán, S., Cortés, A. J., \& Richardson, J. E. (2013) Páramo is the world's fastest evolving and coolest biodiversity hotspot. Frontiers in Genetics, 4, 1-7.

Mason, N. W. H., Mouillot, D., Lee, W. G., \& Wilson, J. B. (2005). Functional richness, functional and functional evenness divergence: the primary of functional components diversity. Oikos, 111, 112-118.

Meneses-Ortegón, L., \& Herrera-Martínez, Y. (2015). Bacterioplancton de tres humedales altoandinos de la Cordillera Oriental de Colombia. Biota Colombiana, 16(1), 1-10.

Millán, A. (2016). Parque Nacional Natural Chingaza. Bogotá D.C., Colombia.

Oksanen, J., Blanchet, F. G., Kindt, R., Legendre, P., Minchin, P. R., O'hara, R. B., ... \& Oksanen, M. J. (2013). Package 'vegan'. Community ecology package, version, 2(9).

Ospina, T. R., Riss, W., \& Ruiz, J. L. (1999). Guía para la identificación genérica de larvas de quironómidos (Diptera: Chironomidae; Orthocladiinae) de la Sabana de Bogotá. In G. Amat, G. Andrade, \& F. Fernández (Eds.), Insectos de Colombia (1a ed., Vol. 2, pp. 363-384). Colombia: Academia Colombiana de Ciencias Exactas, Físicas y Naturales.

Pinder, L. C. V. (1995). The habitats of chironomid larvae. In P. D. Armitage, P. S. Cranston, \& L. C. Pinder (Eds.), The Chironomidae: Biology and ecology of non-biting midges (1a ed., pp. 107-135). UK: Springer.

Pinho, L. C. (2008). Diptera. In Guia on-line: Identificação de larvas de insetos aquáticos do estado de São Paulo (p. 20).

Posada-García, J. A., Abril-Ramírez, G., \& Parra-Sánchez, L. N. (2008). Diversity of aquatic macroinvertebrates of Páramo de Frontino (Antioquia, Colombia). Caldasia, 30(2), 441-455.

Prat, N., González-Trujillo, J. D., \& Ospina-Torres, R. (2014). Clave para la determinación de exuvias pupales de los quironómidos (Diptera: Chironomidae) de ríos altoandinos tropicales. Revista de Biología Tropical, 62(4), 1385-1406.

Prat, N., Rieradevall, M., Acosta, R., \& Villamarín, C. (2011). Guia para el reconocimiento de las larvas de Chironomidae (Diptera) de los ríos altoandinos de Ecuador y Perú: clave par la determinación de los géneros. Barcelona: Grupo de Investigación F. E. M. Departamento de Ecología, Universidad de Barcelona.
R, C. T. (2016). R: A language and Environment for statistical computing. Vienna, Austria: R Foundation for Statistical Computing.

Rosi-Marshall, E. J., Wellard, H. A., Hall, R. O., \& Vallis, K. A. (2016). Methods for quantifying aquatic macroinvertebrate diets. Freshwater Science, 35(1), 229-236.

Ruiz-Moreno, J. L., Ospina-Torres, R., \& Riss, W. (2000). Guía para la identificación genérica de larvas de quironómidos (Diptera: Chironomidae) de la sabana de Bogotá. II. Subfamilia Chironominae. Caldasia, 22(1), 15-33.

Ruiz-Moreno, J., Ospina-Torres, R., Gómez-Sierra, H., \& Riss, W. (2000). Guía para la identificación genérica de larvas de quironómidos (Diptera: Chironomidae) de la sabana de Bogotá. III. Subfamilias Tanypodinae y Diamesinae. Caldasia, 22(1), 34-60.

Sanchez-Hernandez, J., Cobo, F., \& Amundsen, P.-A. (2015). Food web topology in high mountain lakes. PLoS ONE, 10(11), 1-17.

Sanseverino, A. M., \& Nessimian, J. L. (2008). The food of larval Chironomidae (Insecta, Diptera) in submerged litter in a forest stream of the Atlantic Forest (Rio de Janeiro, Brazil). Acta Limnologica Brasiliensia, 20(1), 15-20

Schmid-Araya, J., \& Schmid, P. (1995). Preliminary results on diet of stream invertebrate species: the meiofaunal assemblages. Jber Biological Station Lunz, 15, 23-31.

Schmidt-Mumm, U., \& Ríos, O. V. (2012). Comunidades vegetales de las transiciones terrestre-acuáticas del páramo de Chingaza, Colombia. Revista de Biología Tropical, 60(1), 35-64.

Silva, F. L. (2016). Alotanypus wilhelmensis sp. nov., a new Tanypodinae (Diptera: Chironomidae) from high mountain lakes in Papua New Guinea. Austral Entomology, 55(4), 360-365.

Silva, F. L., Mayer, G., Biscalquini, J. L., \& Silveira, S. (2009). Categorização funcional trófica das comunidades de macroinvertebrados de dois reservatórios na região Centro-Oeste do Estado de São Paulo, Brasil. Maringá, 31(1), 73-78.

Silva, F. L., Ruiz, S. S., Bochini, G. L., \& Moreira, D. C. (2008). Functional feeding habits of Chironomidae larvae (Insecta, Diptera) in a lotic system from Midwestern region of São Paulo State, Brazil. PanAmerican Journal of Aquatic Sciences, 3(2), 135-141.

Tokeshi, M. (1995). The Chironomidae: biology and ecology of non-biting midges. In P. D.

Armitage, P. S. Cranston, \& L. C. V. Pinder (Eds.), Production Ecology (pp. 269-296). London: Springer. 
Tomanova, S., Goitia, E., \& Helesic, J. (2006). Trophic levels and functional feeding groups of macroinvertebrates in neotropical streams. Hydrobiologia, $556,251-264$.

Van Colen, W. R., Mosquera, P., Vanderstukken, M., Goiris, K., Carrasco, M.-C. Decaestecker, E., Alonso, M., ... Muylaert, K. (2017). Limnology and trophic status of glacial lakes in the tropical Andes (Cajas National Park, Ecuador). Freshwater Biology, 62(3), 458-473.
Van der Hammen, T., \& Cleef, A. M. (1986). Development of the high Andean páramo flora and vegetation. En F. Vuilleumier \& M. Monsaterio (Eds.), High Altitude Tropical Biogeography (pp. 153-201).

Zuñiga, O., Uribe, A., Torres, A. M., Cuero, R., \& Peña, J. (2013). Assessment of the impact of anthropic activities on carbon storage in soils of high montane ecosystems in Colombia. Agronomía Colombiana, 31(1), 112-119. 